04

\title{
Трансформация плоского фронта волны в полусферах из лейкосапфира
}

\author{
() В.Н. Ветров, Б.А. Игнатенков, В.Э. Якобсон
}

Научно-исследовательский и технологический институт оптического материаловедения, Государственный оптический институт им. С.И. Вавилова, 193171 Санкт-Петербург, Россия

e-mail: vasvetrov@mail.ru

Поступила в редакцию 26.04.2017 г.

Разработан алгоритм расчета фронтов обыкновенной и необыкновенной волн после прохождения полусферических деталей из одноосного кристалла. Определено влияние частотной дисперсии $n_{o}$ и $n_{e}$, а также изменения направления оптической оси кристалла на фронт необыкновенной волны в полусферах из лейкосапфира и его пластически деформированного аналога.

DOI: 10.21883/OS.2018.01.45364.99-17

\section{Введение}

Детали из одноосных кристаллов (кварца, фторида магния, лейкосапфира) пользуются стабильно растущим спросом на оптическом рынке в связи с разработкой многоканальных систем наблюдения и управления в видимом и ИК диапазонах длин волн. Плоскопараллельные окна и детали сложной формы из лейкосапфира благодаря уникальному сочетанию оптических и прочностных характеристик материала, а также доступностью крупногабаритных кристаллов высокого оптического качества наиболее востребованы в настоящее время. Однако кристаллооптика деталей сложной формы (полусферы, асферические элементы) требует дополнительных исследований в части трансформации волнового фронта.

Формирование двух волновых фронтов плоскопараллельной пластинкой из одноосного кристалла при падении естественного света в соответствии с принципом Гюйгенса показано авторами $[1,2]$. В монографии [3] рассмотрены три случая при ортогональном падении плоской волны на поверхность пластинки, вырезанной из одноосного кристалла: оптическая ось направлена под углом к поверхности, а также наклонное падение волны на пластинку из положительного и отрицательного одноосных кристаллов с оптической осью, расположенной параллельно границе раздела или плоскости падения. Там же показано, что вектор электрического поля обыкновенной волны перпендикулярен оптической оси кристалла и направлению распространения волны. Вектор электрического поля необыкновенной волны не перпендикулярен волновому вектору, однако ортогонален вектору обыкновенной волны.

Цель работы - исследование трансформации плоского фронта волны в одноосных кристаллах и полусферических деталях из них на примере лейкосапфира и его пластически деформированного аналога при разных длинах волн.

\section{Алгоритм расчета}

В качестве объектов исследования выбрали мениск из монокристалла лейкосапфира $\mathrm{c}$ оптической осью кристалла, направленной параллельно оси симметрии детали, и элементы из пластически деформированного лейкосапфира: мениск [4] и полусферическая линза [5] с конусом (в сечении веером) направлений оптической оси кристалла. Полусферические детали отличаются углом оптической оси кристалла к входящей поверхности детали при отклонении падающей волны от оси симметрии детали, который в работе определяется углом $\gamma$, также характеризующим падающую волну (рис. 1).

Для осесимметричной детали из кристалла правомерно решение задачи в плоскости, проходящей через ось симметрии менисков или линзы, т.е. в главной плоскости кристалла.

На рис. 1 приведены схемы преломления монохроматической волны в полусферических деталях из лейкосапфира и его пластически деформированного аналога в системе координат, принятой при разработке алгоритма расчета фронта обыкновенной и необыкновенной волн.

Уравнение входящей поверхности полусферических деталей в принятой системе координат имеет вид

$$
y_{1}=\sqrt{1-\left(1-x_{1}\right)^{2}},
$$

$y_{1}=y / R, x_{1}=x / R, R-$ радиус входящей поверхности детали.

Угол падения луча $\varphi$ на входящую поверхность детали и угол $p$ между необыкновенным лучом и направлением оптической оси кристалла после преломления луча в заданной точке поверхности детали определяли согласно выражениям

$$
\begin{gathered}
\varphi=\operatorname{arctg}\left(1 / y_{1}^{\prime}\right), \\
p=\operatorname{arctg}\left(\sqrt{\frac{n_{e}^{2}}{n_{o}^{2}\left[\left[\frac{n_{e}^{2}}{\sin ^{2} \varphi}\right]-1\right]}}\right),
\end{gathered}
$$



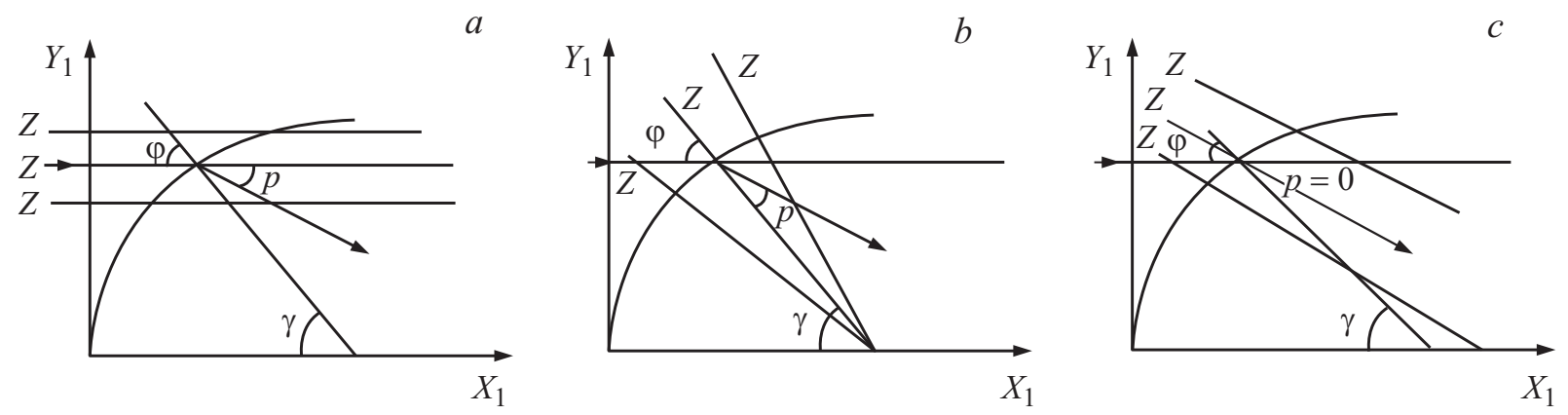

Рис. 1. Схема образования необыкновенной волны на поверхности менисков $(a, b)$ и полусферической линзы $(c)(a-$ из монокристалла лейкосапфира, $b, c$ - из модифицированного лейкосапфира; $p$ - угол между необыкновенной волной и оптической осью кристалла $Z, \gamma-$ угол, характеризующий отклонение падающей волны от оси симметрии детали).

$$
\begin{aligned}
p= & \operatorname{arctg}\left[\left[\left(\frac{1}{\rho n_{o}^{2}-1}\right)\right.\right. \\
& \left.\times\left(\tan \alpha-\sqrt{\tan ^{2} \alpha-\left(\rho n_{0}^{2}-1\right)\left(\rho n_{e}^{2}-\tan ^{2} \alpha\right)}\right)\right],
\end{aligned}
$$

где $n_{o}, n_{e}-$ показатели преломления обыкновенной и необыкновенной волн, $\rho=\sin ^{2} \varphi / n_{o}^{2} n_{e}^{2} \cos \alpha$, $\alpha=\arcsin \varphi$. Последнее выражение является решением универсального уравнения для определения угла между направлением необыкновенной волны и оптической осью кристалла [6] и применимо для полусферической линзы и мениска из монокристалла. Далее определяли $n_{e}(\gamma)$ - локальный показатель преломления необыкновенной волны для точки входящей поверхности детали согласно

$$
n_{e}(\gamma)=n_{o}^{2} n_{e}^{2} / \sqrt{n_{o}^{2} \sin ^{2} p+n_{e}^{2} \cos ^{2} p}
$$

Условно разделим оптический путь волны на три участка.

Расстояние $L_{1}$ до входящей поверхности детали для волны и расстояние $L_{3}$ от вогнутой поверхности детали оценивали как прямолинейное распространение света в вакууме. Траекторию волны $L_{2}$ в детали для каждой точки поверхности детали определяли с учетом изменения оптического пути и скорости распространения для необыкновенной волны. Для определения фронта обыкновенной и необыкновенной волн при разных длинах волн излучения использовали соотношения

$$
\begin{aligned}
& L_{o}=L 1_{o}+L 2_{o}+L 3_{o}, \\
& L_{e}=L 1_{o}+L 2_{e}+L 3_{o},
\end{aligned}
$$

где $L_{o}, L_{e}-$ фронты обыкновенных и необыкновенных волн. В работе фронт необыкновенной волны характеризовали соотношением $\left(L_{e}-L_{o}\right) / \lambda$, где $\lambda-$ длина волны излучения. Так как для лейкосапфира $n_{o}>n_{e}$, то скорость необыкновенной волны больше, и параметр $\left(L_{e}-L_{o}\right) / \lambda \geq 0$.
Скорость обыкновенной волны равна $c / n_{o}(c-$ скорость света) независимо от направления распространения волны. Из элементарных тригонометрических соотношений определяли расстояние, прошедшее излучением со скоростью $c$ от начала отчета: момент достижения плоским волновым фронтом центральной точки детали $(\gamma=0)$. Фронты обыкновенной и необыкновенной волн определяли для момента достижения нецентральной обыкновенной волной $\left(\gamma=60^{\circ}\right)$ вогнутой поверхности детали (рис. 1).

Разработанный алгоритм позволяет учесть для заданной длины волны изменение:

- направления волнового вектора падающей волны,

- скоростей распространения волн,

- оптических путей обыкновенной и необыкновенной волн в детали при прямолинейном распространении волн в деталях.

\section{Обсуждение результатов}

В плоскопараллельной пластинке из одноосного кристалла обыкновенная и необыкновенная волны отличаются линейной поляризацией перпендикулярно главному сечению или в плоскости главного сечения кристалла соответственно. Плоский волновой фронт при прохождении плоскопараллельной пластинки из одноосного кристалла, в которой оптическая ось кристалла наклонена к поверхности, превращается во фронт обыкновенной и необыкновенной волн, в которых сохраняется поляризация волн, так как плоскость главного сечения кристалла не изменяется.

На выходе из пластинки одноосного кристалла имеем два плоских волновых фронта, расхождение между которыми определяется длиной волны излучения и углом между направлением оптической оси кристалла и поверхностью пластинки при падении плоского фронта волны.

При падении плоского фронта волны на полусферическую деталь из одноосного кристалла для оценки изменения волнового фронта необходимо учитывать не только фактор формы детали, учтенный переменными $L 1_{o}$ и 

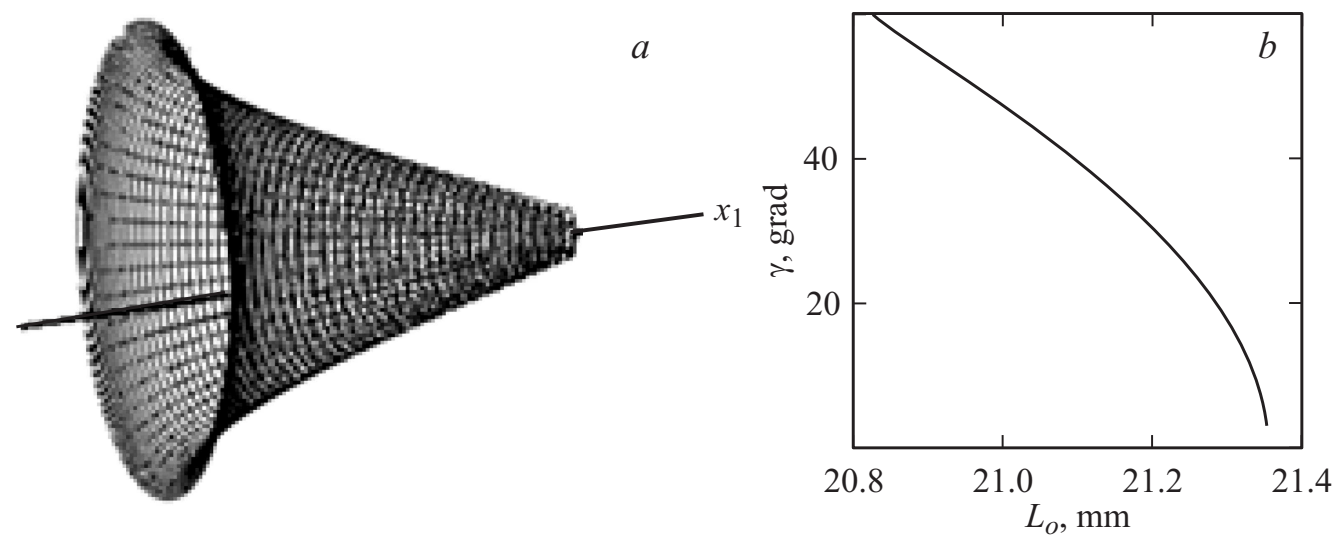

Рис. 2. Фронт обыкновенной волны $(a)$ и его сечение $(b)$ в плоскости, проходящей через ось симметрии детали, после полусферических деталей из лейкосапфира $(R=32 \mathrm{~mm}, h=4 \mathrm{~mm}, \lambda=4.206 \mu \mathrm{m})$.

$L 3_{o}$, а также изменение оптического пути волн и скорости распространения необыкновенной волны в детали.

Особенность деталей из пластически деформированного лейкосапфира: в каждой точке поверхности детали имеем свое главное сечение кристалла, что позволяет при наличии конуса оптических осей в детали получить осесимметричную (круговую) поляризацию волн относительно оси симметрии детали, а в плоскости, проходящей через эту ось, поляризация необыкновенной волны обусловлена изменением положения главного сечения деформированного кристалла. Поляризация обыкновенной волны находится в плоскости рассматриваемого сечения (рис. 1) и перпендикулярна направлению ее распространения.

Изменение фронта обыкновенной волны после преломления на поверхности деталей в любом из рассматриваемых случаев (рис. 1) равна $c / n_{o}(\lambda)$ независимо от направления оптической оси кристалла. Волна, падающая вдоль оптической оси кристалла, направленной по оси симметрии детали, распространяется по наикратчайшему пути. Для других волн оптический путь увеличивается с увеличением угла $\gamma$, на выходе имеем волновой фронт, осесимметричный относительно оси симметрии детали (рис. 2).

При увеличении длины волны излучения изменяется угол преломления луча, обусловливающий изменение оптического пути обыкновенной волны в детали, а также увеличивается скорость его распространения, поэтому вдоль оси симметрии изменение волнового фронта наибольшее (рис. 3, кривые 1,2).

При достижении фронтом обыкновенной волны внутренней поверхности полусферической детали $\left(\gamma=60^{\circ}\right.$, рис. 3), т.е. на выходе из детали, волновой фронт имеет форму параболы с максимально удаленной точкой от поверхности на оси симметрии детали. Частотная дисперсия $n_{o}$ (при увеличении длины волны излучения) также обусловливает наибольшее увеличение пути волны в центральной точке волнового фронта. Так, при изменении длины волны с $\lambda=0.535 \mu \mathrm{m}$ на $\lambda=4.206 \mu \mathrm{m}$ разность, отнесенная к последней длине волны излучения, равна $30.21 \lambda$.

Фронт необыкновенных волны, выраженный в долях длины волны излучения, сдвинут по координате $x_{1}$ в сторону больших значений.

Наибольшее расхождение волновых фронтов после прохождения детали наблюдается на периферии менисков, причем для детали из монокристаллического лейкосапфира (рис. 3 ) составляет $\sim 1.5 \lambda$. В центральной точке фронты волн совпадают и их изменения, обусловленные частотной дисперсией показателей преломления, равнозначны, так как скорости распространения лучей вдоль оптической оси кристалла равны. В полусферической линзе из пластически деформированного лейкосапфира [5] получен такой же результат: при изменении длины волны излучения (рис. 4) изменения фронтов обыкновенной и необыкновенной волн подобны.

Итак, частотная дисперсия показателей преломления обусловливает максимальное изменение фронта обыкновенной волны в центре деталей.

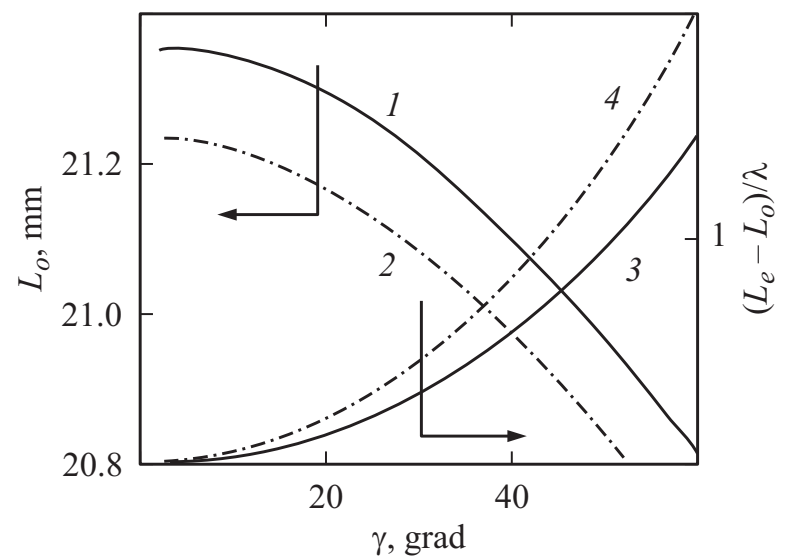

Рис. 3. Сечения фронта обыкновенной волны $(1,2)$ и изменения фронта необыкновенной волны $(3,4)$ в мениске из монокристаллического лейкосапфира $(\lambda=4.206 \mu \mathrm{m}(1,3)$ и $0.535 \mu \mathrm{m}(2,4))$. 


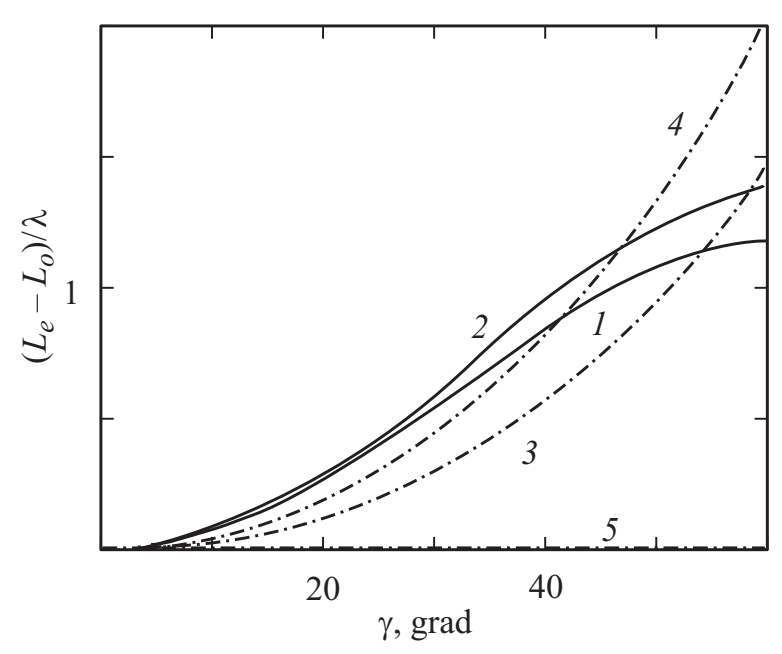

Рис. 4. Приращение фронтов необыкновенных волн в деталях из лейкосапфира $(1,2-$ мениск из пластически деформированного лейкосапфира, 3,4- мениск из монокристалла, $5-$ полусферическая линза; $\lambda=4.206 \mu \mathrm{m}(1,3,5)$ и $0.535 \mu \mathrm{m}(2,4,5))$.

Изменение фронтов необыкновенных волновых в деталях, приведенное на рис. 4, показывает, что разворот оптических осей кристалла при пластической деформации снижает изменение фронта волны на краю мениска по сравнению с деталью из монокристалла. В полусферической линзе расхождение волновых фронтов отсутствует: после преломления на поверхности детали распространение волн происходит вдоль оптической оси кристалла, т.е. подобно лучу, проходящему по оси симметрии детали [6]. После полусферической линзы наблюдаем один фронт волны по всей апертуре детали, который имеет параметры фронта обыкновенной волны. При уменьшении длины волны излучения наиболее изменяется фронт волны мениска из монокристалла в периферийной области. В полусферической линзе из модифицированного лейкосапфира изменение фронта необыкновенной волны на краю детали менее $0.015 \lambda$. Сравнение влияния частотной дисперсии показателей преломления на фронт волны в полусферических деталях из лейкосапфира показывает, что в полусферической линзе из лейкосапфира ее влияние наименьшее, т. е. двоякодисперсионность, присущая одноосным кристаллам, очевидно, не будет иметь значения [7].

\section{Выводы}

В заключение можно сделать следующие выводы: изменение показателя преломления необыкновенных лучей при отклонении от центра полусферических деталей из лейкосапфира обусловливает образование двух волновых фронтов на выходе из детали с общей точкой на оси симметрии детали. Направление оптической оси кристалла относительно поверхности детали определяет фронт необыкновенных волн. В деталях из пластически деформированного лейкосапфира приращение фронта необыкновенных волн меньше на краю мениска и по всей апертуре для полусферической линзы.

Разработанный алгоритм расчета волновых фронтов лучей позволяет априори оценить их параметры после трансформации в деталях сложной формы из одноосных кристаллов, а также определить направления распространения обыкновенной и необыкновенной волн после детали.

\section{Список литературы}

[1] Шустер A. Введение в теоретическую оптику. Л., М.: ОНТИ, 1935. $376 \mathrm{c.}$

[2] Шредер Г., Трайберг X. Техническая оптика. М.: Техносфеpa, 2006. $424 \mathrm{c}$.

[3] Ярив А., Юх П. Оптические волны в кристаллах. М.: Мир, 1987. $616 \mathrm{c}$.

[4] Афбанасьев И.И., Андрианова Л.К., Ветров В.Н., Игнатенков Б.А., Рыжиков Э.Н. Патент РФ № 17739561993.

[5] Ветров В.Н., Игнатенков Б.А., Письменный В.А., Рыжиков Э.Н., Дукельский К.В. Патент РФ № 23776142009.

[6] Ветров В.Н., Игнатенков Б.А. // Опт. и спектр. 2009. T. 106. № 1. C. 154158.

[7] Афанасьев И.И., Андрианова Л.К. // ОМП. 1991. № 10. C. $30-33$. 